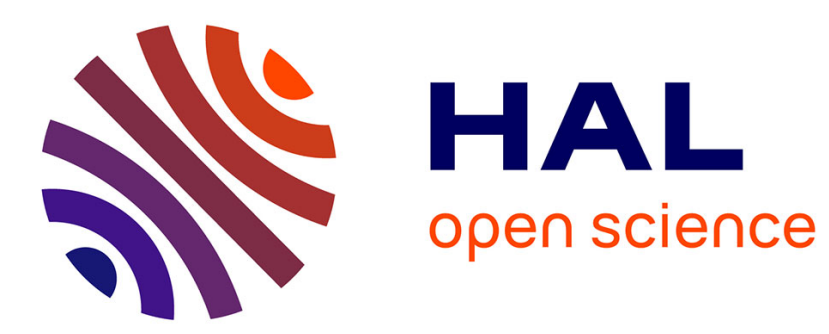

\title{
Electric-field effect on coercivity distributions in FePt magneto-electric devices
}

Liza Herrera-Diez, Anne Bernand-Mantel, Ondra Michele, Laurent Vila, Patrick Warin, Alain Marty, Laurent Ranno, Dominique Givord

\section{To cite this version:}

Liza Herrera-Diez, Anne Bernand-Mantel, Ondra Michele, Laurent Vila, Patrick Warin, et al.. Electric-field effect on coercivity distributions in FePt magneto-electric devices. Applied Physics Letters, 2013, 102, pp.012409. 10.1063/1.4774382 . hal-00773419

\section{HAL Id: hal-00773419 \\ https://hal.science/hal-00773419}

Submitted on 14 Jan 2013

HAL is a multi-disciplinary open access archive for the deposit and dissemination of scientific research documents, whether they are published or not. The documents may come from teaching and research institutions in France or abroad, or from public or private research centers.
L'archive ouverte pluridisciplinaire HAL, est destinée au dépôt et à la diffusion de documents scientifiques de niveau recherche, publiés ou non, émanant des établissements d'enseignement et de recherche français ou étrangers, des laboratoires publics ou privés. 


\section{Electric-field effect on coercivity distributions in FePt magneto-electric devices}

L. Herrera Diez, ${ }^{1, a)}$ A. Bernand-Mantel, ${ }^{1}$ O. Michele, ${ }^{1}$ L. Vila, ${ }^{2}$ P. Warin, ${ }^{2}$ A. Marty, ${ }^{2}$ L. Ranno, ${ }^{1}$ and D. Givord ${ }^{1}$

1) Institut Néel - CNRS-UJF, 25 avenue des Martyrs, F-38042 Grenoble Cedex 9, France

2) INAC/SP2M CEA-UJF, 17 avenue des Martyrs, F-38054 Grenoble,

France

We have investigated the contribution of stochastic thermally activated processes to the electric-field effects on coercivity in FePt. Coercive field distributions were measured under different gate voltages in solid-state field-effect structures. For low voltages a shift in the coercive field distribution can be observed, however, it is not larger than the width of the distribution. Higher voltages are needed to obtain the splitting from the negative (zero) voltage distribution allowing for the unambiguous characterization of the electric-field effect. A virtual unipolarity in the electric-field effect has been identified as a feature introduced by the dielectric layer that disappears upon annealing.

Keywords: FePt, electric-field effect, magnetic anisotropy.

a)Electronic mail: liza.herreradiez@grenoble.cnrs.fr 
The electric field (E) control of magnetic properties already demonstrated in a variety of itinerant ferromagnets ${ }^{1-6}$ constitutes an attractive path towards low power magnetization switching applications. In FePt, the reduction of the coercive field observed under electron charging was attributed to a reduction in the large magnetocrystalline anisotropy of this compound, resulting from the voltage-induced $3 \mathrm{~d} / 5 \mathrm{~d}$ band filling ${ }^{1,7}$. In Ref. [1], the change in the electron density was obtained by creating an electric field between the sample and a so-called counter-electrode, both immersed in a liquid electrolyte. The large dielectric constant of the so-called double-layer formed at the sample surface permitted significant charging upon applying a moderate voltage below $1 \mathrm{~V}$.

With the prospect of exploiting the E-control of magnetism in magneto-electric devices, its observation in structures incorporating a solid-state barrier appears as a prerequisite. This is realized in the present study, where the electric field is generated across an insulating $\mathrm{HfO}_{2}$ barrier, deposited on top of a $2 \mathrm{~nm} L 1_{0}$ epitaxial FePt film. In these devices the measured coercive field is found to fluctuate by typically 1-2 \% upon repeating the experiment giving rise to a coercive field distribution. This feature was already observed in a number of studies dealing with FePt ultra-thin films ${ }^{8,9}$ and can be ascribed to the stochastic nature of the thermal activation processes involved in overcoming the coercive energy barrier associated to a small number of events. It is worth mentioning that in the cited studies the FePt films were patterned into Hall bar structures but were not covered with a dielectric layer as in the present case. This acertains that the coercive field distribution does not arise from the creation of a $\mathrm{FePt} / \mathrm{HfO}_{2}$ interface, necessary to build the field-effect devices discussed in this study.

The width of the coercive field distribution may mask the modification of the coercive field induced by the applied E-field and therefore the impact of stochastic thermally activated processes needs to be addressed. In this study we present the effect of electric fields generated in top-gate electrode devices on the coercivity distributions of thin films of FePt at various gate voltages.

The magnetic films used in this work consist of a $2 \mathrm{~nm}$ thick epitaxial FePt $L 1_{0}$ layer grown on $\mathrm{Pt}(001)(30 \mathrm{~nm}) / \mathrm{Cr}(2 \mathrm{~nm}) / \mathrm{MgO}(001)^{10}$. The films were patterned into Hall bar structures which have a 50x450 $\mathrm{m}^{2}$ main channel. Subsequently, a $\approx 10 \mathrm{~nm}$ thick dielectric layer of $\mathrm{HfO}_{2}$ was deposited by atomic layer deposition at a temperature of $150^{\circ} \mathrm{C}$ on the entire magnetic structures. $\mathrm{HfO}_{2}$ was chosen as the dielectric material due to its high 
dielectric constant (nominally 25) which makes it an extensively used material in field-effect structures where a large electric polarization inside the insulating barrier is needed ${ }^{12}$. In a last step, the top gate $\mathrm{Au}$ (50 nm thick) electrode was deposited covering an area of $10^{4}$ $\mu \mathrm{m}^{2}$ over the main Hall bar channel. Fig. 1 shows a graphic representation of the structure of the devices. All hysteresis loops were obtained by extraordinary Hall effect measurements at room temperature using a DC bias current of $1 \mathrm{~mA}$ and a constant magnetic field rate of $0.1 \mathrm{~T} / \mathrm{min}$ (saturation field $=0.25 \mathrm{~T}$ ). The resulting current density is $6 \times 10^{8}$ $\mathrm{A} / \mathrm{m}^{2}$, well below the reported current values for which Joule heating needs to be taken into account ${ }^{13}$. The voltage drop along the Hall bar main channel when passing a current of $1 \mathrm{~mA}$ was approximately $50 \mathrm{mV}$ (i.e. resistance $=50 \Omega$ ), significantly smaller than the gate voltages applied to the top electrode. The coercive field (defined as the point of zero net magnetization) was of the order of 0.13-0.15 $\mathrm{T}$ at room temperature, a value similar to the one reported in [1] for a continuous film as well as in several recent studies ${ }^{2,3}$. It is worth noting that the FePt films used in these experiment are grown in the same facilities and in the same conditions as those from reference [1], however, in this study the measured area is more than one order of magnitude smaller. This explains the much more prominent role of individual thermally activated processes leading to the appearance of a coercive field distribution in the present case compared to the E-field measurements in [1]. All coercivity distributions have been obtained from a number of Hall effect loops ranging from 25 to 30 which represents a measurement time of about two hours for each given gate voltage.

Fig. 2 (a), (b), (c) and (d) present the coercive field distributions at gate voltages of \pm $1 \mathrm{~V}, 2 \mathrm{~V}, 3 \mathrm{~V}$ and $4 \mathrm{~V}$, respectively. The measurements in Fig. 2 have been carried out on a single magneto-electric device and the behavior observed has been reproduced in a number of equivalent devices. For the sake of clarity, it is worth mentioning that the gate voltages informed refer to the voltage applied on the Au gate electrode. Therefore, a positive gate voltage translates into the creation of negative charges on the counter FePt electrode, i.e. filling of the electronic band states of FePt and vice versa. The two distributions measured under gate voltages of $\pm 1 \mathrm{~V}$ are almost superimposed, only at gate voltages of $\pm 2 \mathrm{~V}$ a visible splitting of the distributions for negative and positive gate voltages is observed (Fig. $2(\mathrm{~b})$ ). The splitting continues to increase at higher voltages but it is only for $\pm 4 \mathrm{~V}$ that the overlapping between the two distributions completely disappears.

These observations illustrate the above-mentioned stochastic character of the thermally 

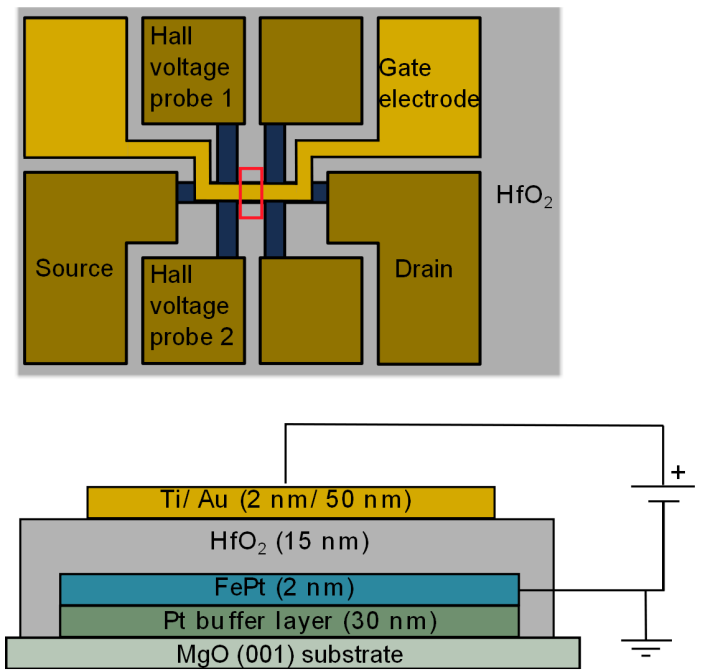

FIG. 1. Structure of the magnetic field-effect devices. The patterned Hall bar structure and the position of the gate electrode are shown in the top part of the sketch. The magnetic film consists of a $2 \mathrm{~nm}$ thick epitaxial FePt $L 1_{0}$ layer grown on $\mathrm{Pt}(001)(30 \mathrm{~nm}) / \mathrm{Cr}(2 \mathrm{~nm}) / \mathrm{MgO}(001)^{10}$. After patterning a dielectric $10 \mathrm{~nm} \mathrm{HfO}_{2}$ layer was deposited followed by the deposition of the top gate $\mathrm{Au}(50 \mathrm{~nm})$ electrode.

a)

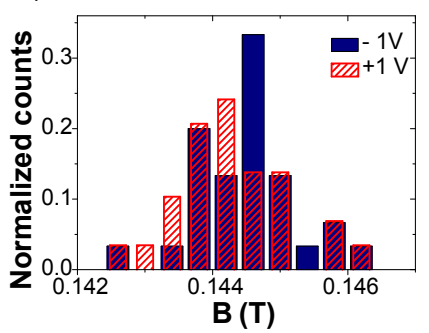

c)

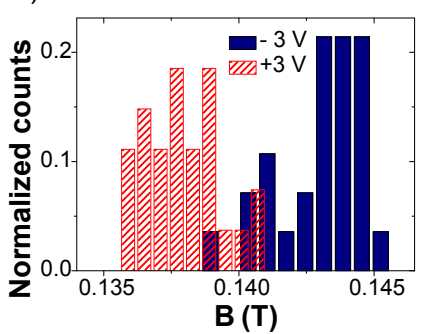

b)

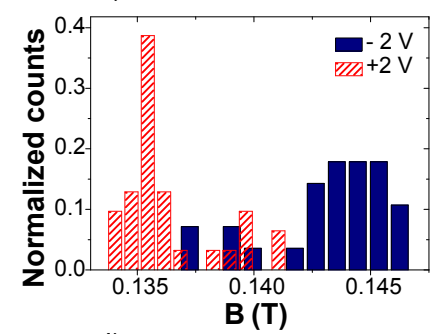

d)

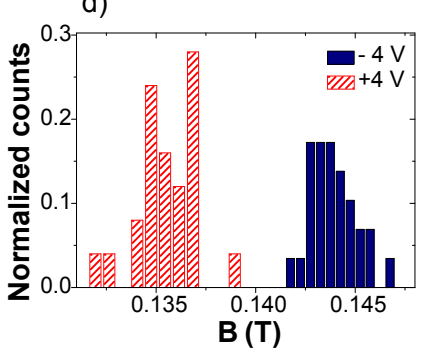

FIG. 2. Coercivity distributions measured under gate voltages of (a) $\pm 1 \mathrm{~V},(\mathrm{~b}) \pm 2 \mathrm{~V},(\mathrm{c}) \pm 3 \mathrm{~V}$ and $(\mathrm{d}) \pm 4 \mathrm{~V}$. The overlapping between the distributions at positive and negative gate voltages disappears for $\pm 4 \mathrm{~V}$. 
activated processes involved in the magnetization reversal in FePt which are ultimately responsible for the existence of a coercive field distribution ${ }^{8,9}$. The observed partial overlapping of the coercive field distributions does not mask the action of the electric field on coercivity. However, in the presence of a coercive field distribution the existence of the E-effect can only be established unambiguously via repeating the measurements as done here.

The distributions at positive gate voltages appear at lower field values than those corresponding to negative gate voltages of the same magnitude. This indicates that the coercive field value is reduced in the presence of a positive gate voltage. This reduction in the coercive field can be interpreted as a weakening of the magnetic anisotropy due to band filling. This is in agreement with field-effect measurements on epitaxial FePt films in contact with a liquid electrolyte ${ }^{1}$ as well as with electronic structure calculations ${ }^{7}$. Although demonstrating E-effects in this system, these measurements also reveal that the coercive field under negative voltage, up to the maximum value of $-4 \mathrm{~V}$, is approximately identical to the one measured under zero applied voltage, namely, that there is an asymmetry in the effect of the applied gate voltage. This can be seen in Fig. 3 (a) showing the same $\pm 4 \mathrm{~V}$ distributions as in Fig. 2 (d) together with a 0V distribution, this asymmetry is also shown in Fig. 3 (b) which presents Hall effect hysteresis loops taken under gate voltages of 0V, $+4 \mathrm{~V}$ and $-4 \mathrm{~V}$. A similar behaviour has been reported in Fe based field effect structures and attributed to a large charge trapping effect inside the $\mathrm{ZrO}_{2}$ dielectric layer ${ }^{11}$. It is well known that materials with high dielectric constants such as $\mathrm{HfO}_{2}$ and $\mathrm{ZrO}_{2}$ present a tendency for charge trapping that compromises their performance in field-effect devices ${ }^{12,14,15}$. This led us to ascribe the asymmetric voltage effect to such charge trapping effects. The charge trapping effects in $\mathrm{HfO}_{2}$ have been related to structural defects in the dielectric such as oxygen vacancies and have been shown to be strongly dependent on the composition of the gate electrodes ${ }^{16}$ and their deposition method ${ }^{17}$. The asymmetry in the E-field effect may be explained by considering that the electronic structure in the vicinity of the trap (defect) sites in $\mathrm{HfO}_{2}$ defines an affinity for the trapping of charges of a given sign. In this context the E-field induced charge trapping would preferentially occur under one of the two possible signs of the gate voltage. This scenario would translate into an asymmetric effect of the gate voltage on the magnetic properties of the FePt films similar to what has been reported in Ref[17]. This description is supported by studies suggesting that oxygen vacancies in $\mathrm{HfO}_{2}$ 
a)

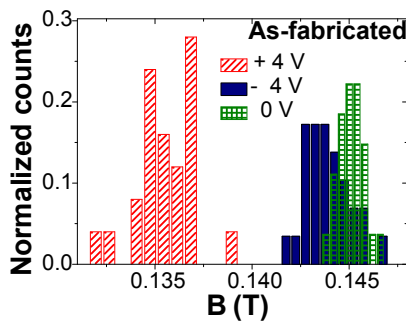

c)

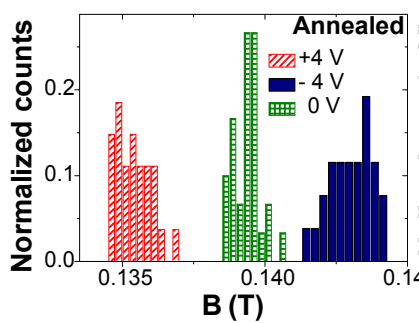

b)

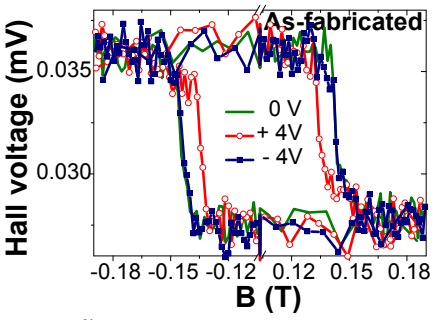

d)

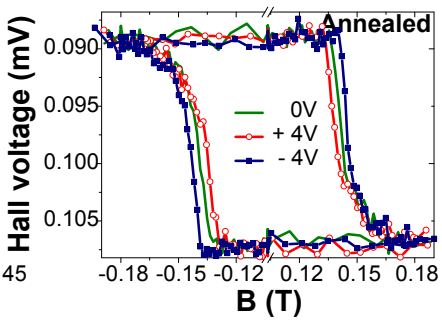

FIG. 3. Coercivity distributions and hysteresis loops measured under gate voltages of $\pm 4 \mathrm{~V}$ and $0 \mathrm{~V}$ in as-fabricated $(\mathrm{a}, \mathrm{b})$ and annealed $(\mathrm{c}, \mathrm{d})$ devices. The annealing process eliminates a virtual unipolarity of the gate voltage effect on the value of the coercive field.

can be intrinsic electron traps presenting a variety of ionization states ${ }^{18}$.

Along the same lines, annealing in an $\mathrm{O}_{2}$ rich environment has been shown to significantly reduce the number of oxygen vacancies ${ }^{19}$ in $\mathrm{HfO}_{2}$ MOSFETs with an associated reduction in the charge trapping effect. This has been confirmed in our devices after annealing in air at a temperature of $200{ }^{\circ} \mathrm{C}$ for two hours. Fig. 3 (c) shows measurements after the annealing treatment where the coercive field distributions measured under positive and negative voltages became symmetrically displaced with respect to the zero voltage coercive field distribution. This is also evident in the hysteresis loops shown in Fig. 3 (d).

The charge trapping effects discussed above provide a simple explanation for the results presented in this study, however, the exact nature of the charge trapping mechanism remains an open question and requires additional studies. It will be particularly interesting to investigate these processes not only to avoid charge trapping in variable gate voltage operation but also to explore the possibility of using them as a route to permanent E-field effects without the need of a constant gate voltage. This concept has been recently investigated in magnetic structures containing a dielectric layer with optically induced trapped charges ${ }^{11}$.

In the annealed sample the relative shift of the coercive field obtained for either positive or negative gate voltages amounts to $0.7 \% / \mathrm{V}$. The field effect reported for FePt immersed 
in a liquid electrolyte ${ }^{1}$ was of $7.6 \% / \mathrm{V}$ for a coercivity similar to that of the present film $(0.115 \mathrm{~T})$. In the cited work, the estimated capacitance is of $30 \mu \mathrm{F} / \mathrm{cm}^{2}$ while in this study the measured capacitance is between 2.3 and $2.8 \mu \mathrm{F} / \mathrm{cm}^{2}$ which accounts for the differences observed in the E-field assisted coercivity changes per applied Volt. This confirms that the magnitude of the effect observed in a liquid electrolyte environment can be also achieved in a solid state device allowing for bipolar operation, a feature that could not be implemented in Ref.[1] due to redox potential restrictions.

It is worth mentioning that the nature of the metal/dielectric interface has been shown to be of great importance to the E-field modulation of the magnetic properties. The fabrication of the devices presented in this study has been carried out without a capping layer protecting the FePt film from air, however, other studies in the literature reported that under similar conditions the E-field effect is absent and that the observation of a reduced $0.1 \% / \mathrm{V}$ effect requires the presence of a protective in-situ grown $\mathrm{MgO}$ layer ${ }^{20}$. In addition, the E-field induced changes in $\mathrm{H}_{c}$ informed in Ref.[18] seem to have the opposite dependence on the sign of the gate voltage as those presented here.

In conclusion, the E-field changes in coercivity observed in Ref. [1] have been brought into a solid state device. Using these structures we have studied E-field effects in the coercivity distribution of FePt. Stochastic thermally activated processes taking part in the magnetization reversal tend to mask the gate-voltage induced coercivity changes in a low gate-voltage range. The electric-field effect on coercivity was first observed at a gate voltage of $\pm 2 \mathrm{~V}$, however, the overlap between the coecivity distributions for positive and negative gate voltages disappears only at $\pm 4 \mathrm{~V}$. A virtual unipolar effect of the gate voltage has been observed and attributed to a charge trapping effect in the $\mathrm{HfO}_{2}$ dielectric layer. This can be removed by a simple annealing treatment in order to improve the devices performance when variable gate voltage operation is needed. At the same time we foresee charge trapping effects in the dielectric layer as a possible route to non-volatility of the E-field effect in magnetic structures. 


\section{ACKNOWLEDGMENTS}

We gratefully acknowledge funding from the French National Research Agency (ANR) under the project ANR-2010-BLAN-1006-ELECMADE and from the Nanosciences Foundation of Grenoble. O. M. acknowledges financial support from the UJF-Brno University of Technology under the SOCRATES program. Device fabrication was carried out at the Nanofab cleanroom facilities in Grenoble.

\section{REFERENCES}

${ }^{1}$ M. Weisheit, S. Fähler, A. Marty, Y. Souche, C. Poinsignon, D. Givord, Science 315, 349-351 (2007).

${ }^{2}$ T. Maruyama, Y. Shiota, T. Nozaki, K. Ohta, N. Toda, M. Mizuguchi, A. A. Tulapurkar, T. Shinjo, M. Shiraishi, S. Mizukami, Y. Ando, Y. Suzuki, Nat. Nano. 4, 158-161 (2009). ${ }^{3}$ M. Endo, S. Kanai, S. Ikeda, F. Matsukura, H. Ohno, Appl. Phys. Lett. 96, 212503 (2010).

${ }^{4}$ D. Chiba, S. Fukami, K. Shimamura, N. Ishiwata, K. Kobayashi, T. Ono, Nat. Mater. 10, 853856 (2011).

${ }^{5}$ W.-G. Wang, M. Li, S. Hageman, C. L. Chien, Nat. Mater. 11, 64-68 (2012).

${ }^{6}$ F. Bonell, S. Murakami, Y. Shiota, T. Nozaki, T. Shinjo, Y. Suzuki Appl. Phys. Lett. 98, 232510 (2011).

${ }^{7}$ G. H. O. Daalderop, P. J. Kelly, M. F. H. Schuurmans, Phys. Rev. B 44, 12054 (1991).

8 J. P. Attané, D. Ravelosona, A. Marty, Y. Samson, C. Chappert, Phys. Rev. Lett. 96, 147204 (2006).

${ }^{9}$ L. Herrera Diez, A. Bernand-Mantel, L. Ranno, D. Givord, L. Vila, P. Warin. A. Marty, New J. Phys. 14, 113024 (2012).

${ }^{10}$ D. Halley, A. Marty, P. Bayle-Guillemaud, B. Gilles, J. P. Attané, Y. Samson, Phys. Rev. $B$ 70, 174438 (2004).

${ }^{11}$ U. Bauer, M. Przybylski, J. Kirschner, G. S. D. Beach, Nano Lett. 12, 1437 (2012).

${ }^{12}$ G. D. Wilk, R. M. Wallace, J. M. Anthony, J. Appl. Phys. 89, 5243 (2001).

${ }^{13}$ A. P. Mihai, F. Garcia-Sanchez, L. Vila, A. Marty, L. D. Buda-Prejbeanu, J. C. Pillet, C. Beigné, D. Ravelosona, C. Mannequin, J. C. Toussaint, J. P. Attané, Phys. Rev. B 84, 014411 (2011). 
${ }^{14}$ N. A. Chowdhury, R. Garg, D. Misraa, Appl. Phys. Lett. 85, 3289 (2004).

${ }^{15}$ H. Jin, M. Chenyue, Z. Lining, Z. Jian, Z. Xing, J. Semicond. 30, 084003 (2009).

${ }^{16}$ K. Xiong, J. Robertson, Microelectronic Engineering 80, 408 (2005).

${ }^{17}$ W. J. Zhu, T. P. Ma, S. Zafar, T. Tamagawa, IEEE Electron Device Lett. 23, 597 (2002).

18 J. L. Gavartin, D. Muñoz Ramo, A. L. Shluger, G. Bersuker, B. H. Lee, Appl. Phys. Lett. 89, 082908 (2006).

${ }^{19}$ M. Jo, H. Park, M. Chang, H.-S. Jung, J.-H. Lee, H. Hwang, Microelectron. Eng. 84, 1934 (2007).

${ }^{20}$ T. Seki, M. Kohda, j. Nitta, K. Takanashi, Appl. Phys. Lett. 98, 212505 (2011). 